\title{
Workflow Considerations in PET/MR Imaging
}

\author{
Gustav K. von Schulthess and Patrick Veit-Haibach \\ Department of Medical Radiology, University Hospital, Zurich, Switzerland
}

\begin{abstract}
Many groups attempt to optimize imaging protocols on PET/MR imaging systems. Although research protocols may take as long as 60-90 min, much more efficient clinical workflows are needed to achieve cost-effective examination times of less than $1 \mathrm{~h}$. Considering these difficulties, simultaneous PET/MR imaging is an intriguing research tool, but its clinical applications are uncertain or just beginning to emerge. However, unlike PET/CT, in which the options for various CT protocols are limited, the MR imaging portion of PET/ MR imaging can be extended arbitrarily depending on the MR pulse sequences chosen. For PET/MR imaging to be complementary, feasible, and somewhat competitive with PET/CT, image acquisition times should ideally be limited to $30 \mathrm{~min}$. The purposes of this article are to help the reader to understand the critical workflow issues in simultaneous PET/MR imaging in comparison with sequential PET/ MR imaging and to learn how to optimize an imaging examination. Current knowledge toward this goal is summarized.
\end{abstract}

Key Words: oncology; PET/MRI; PET/CT-MRI; workflow; imaging protocols

J Nucl Med 2014; 55:19S-24S

DOI: 10.2967/jnumed.113.129239

\section{A}

dual-modality hybrid imaging system differs in critical ways from two separate systems. Among hybrid systems, we distinguish simultaneous and sequential systems $(1,2)$. Sequential imaging systems require a shuttle system that moves patients from one modality to another without changing patient position. SPECT/CT and PET/ CT systems are such sequential systems whereby the scanner bed also serves as the shuttle, and two vendors have introduced this approach for PET/MR imaging. Two manufacturers have introduced true simultaneous PET/MR imaging systems that do not require a shuttle. PET/MR imaging is critically different from PET/ CT and SPECT/CT in that CT data acquisition is fast compared with PET data acquisition, whereas MR data acquisition may be longer than PET data acquisition. Three points are critical to understand in developing PET/MR imaging protocols that provide efficient patient workflow.

First, dual-modality hybrid systems (PET/MR imaging) are typically almost twice as expensive as two single-modality systems. Hence, imaging equipment would be best used by simultaneous

\footnotetext{
Received Nov. 12, 2013; revision accepted Apr. 2, 2014.

For correspondence or reprints contact: Patrick Veit-Haibach, Department of Medical Radiology, Division of Nuclear Medicine, University Hospital, Rämistrasse 100, 8091 Zurich, Switzerland.

E-mail: patrick.veit-haibach@usz.ch

Published online May 1, 2014.

COPYRIGHT (c) 2014 by the Society of Nuclear Medicine and Molecular Imaging, Inc.
}

data acquisition rather than separate acquisitions for MR imaging and PET.

Second, dual-modality systems can save 10-15 min because patient positioning and removal occur only once. The overall time saving is $10-15$ min per examination, provided that both systems acquire data for a comparable length of time.

Third, a dual-modality system is one system. The accuracy of the imaging findings results from the information derived from both systems together.

\section{DUAL-MODALITY PET/MR IMAGING SYSTEMS ARE EXPENSIVE}

Because of the high purchase price of PET/MR imaging, operating such systems efficiently requires image acquisition durations to match those of PET/CT. List prices of such systems are approximately $€ 5$ million, or approximately $\$ 7$ million. As previously stated, dual-modality systems can save approximately 10-15 min per examination.

Because MR imaging acquisitions may take as long as $60 \mathrm{~min}$ and whole-body PET scans may require $15 \mathrm{~min}$, the PET/MR imaging system would be used as an MR imaging scanner $75 \%$ of the time. It may therefore be beneficial to use a shuttle system to increase the parallel use of PET and MR imaging.

Such workflow thoughts may prompt potential PET/MR imaging users to consider purchasing two-room shuttle systems depending on the clinical need. Obviously, in this setting, a large difference in imaging time on both systems is detrimental to an effective workflow. Even in a single room with shuttle-connected PET/MR imaging, the overall imaging time must be kept as short as possible because only one patient can occupy a single room at any time; in such systems, PET and MR imaging times are additive and cannot overlap.

\section{SAVING TIME USING INTEGRATED SYSTEMS}

Any hybrid system has the advantage that the patient has to be uploaded and downloaded from the hybrid scanner only once, a process that takes approximately 10-15 min. Hybrid imaging thus saves approximately $10-15 \mathrm{~min}$, time that can be used for imaging rather than moving the patient. For instance, in a patient with lung cancer, a PET/MR imaging protocol providing similar information to PET/CT could be supplemented by a 10-min contrast-enhanced MR scan of the brain to rule out brain metastasis. The schematic in Figure 1 depicts such data acquisition.

Imaging protocols must be designed to achieve the highest diagnostic accuracy. Because both parts of the imaging system contribute to the accuracy of the test, information from both systems must be optimized, not for each examination individually, but as a whole. In fact, data from both modalities can be complementary, confirmatory, or redundant. 


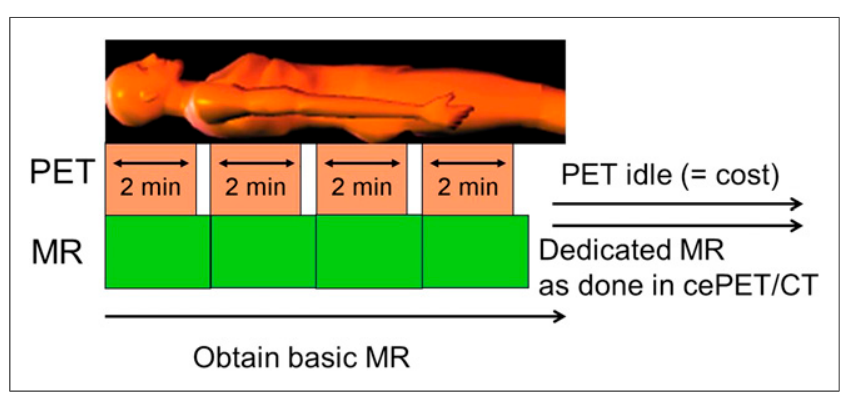

FIGURE 1. Schematic of PET/MR whole-body data acquisition. While PET is being acquired in transaxial field of view of $15-25 \mathrm{~cm}$ for a few minutes, all MR data necessary should be acquired. Adding specific imaging sequences at the end of the PET/MR examination lasting for 10-15 min is still fairly efficient. With longer additional MR imaging times, PET/MR imaging system would be used predominantly as MR imaging system. cePET/CT = contrast-enhanced PET/CT.

Neither SPECT/CT nor PET/CT allows flexible imaging protocols. The options for acquiring complementary and confirmatory rather than redundant data are limited. Some data suggest it is not necessary to acquire contrast-enhanced $\mathrm{CT}$ in many cancers, including breast cancer, lymphoma, and melanoma; moreover, it has not been clearly established that acquiring high-dose versus low-dose CT data improves overall examination specificity (3). However, contrast-enhanced CT in PET/CT increases diagnostic accuracy in some situations, such as liver lesions or other abdominal malignancies. However, adding MR imaging to PET provides the powerful capability of a flexible pulse sequence that can be tailored to acquire a wealth of additional information. The choice of these sequences becomes an essential ingredient for a good workflow, because MR image acquisition times for cost-effective equipment use are limited. As the PET scanner acquires transaxial data for 15- to 25-cm fields of view within 2-5 min, simultaneous MR pulse sequences must fit into this time frame (Fig. 1). Optimized pulse sequences must provide at least as much information as low-dose unenhanced CT studies, which appears to be an achievable goal. However, a low-dose CT protocol would be considered suboptimal in many institutions throughout the world. Therefore, PET/MR imaging studies must be compared with PET/CT studies with and without contrast medium to determine whether PET/MR imaging is inferior, equivalent, or superior to fully diagnostic PET/CT.

Assuming that for some indications MR imaging is better than $\mathrm{CT}$, and that therefore PET/MR imaging is superior to PET/CT, is simplistic. This truth is illustrated in Figure 2, which depicts a patient with extensive liver metastases that are detected by PET alone. Although low-dose unenhanced CT does not detect the lesions, a simple Dixon-type MR pulse sequence shows the metastases. However, the MR imaging information does not add to the information already provided by PET. Here, PET/MR imaging and PET/CT provided the same information, despite the negative CT scan, and the MR data are redundant because the diagnosis of liver metastases can be made by PET alone.

However, simultaneous PET/MR imaging studies may be advantageous to address research questions. Such advantages include measurements of brain activation and blood flow in neurology research. Simultaneous measurements of tumor perfusion and metabolic parameters-for example, during radiation therapy and chemotherapy-may provide noninvasive insights into tumor biology. For a standard clinical work-up, the advantage is not as clear as for research applications because the meaning of simultaneity in a clinical routine is not yet clear. Of course, the greatest disadvantages of PET/MR imaging are the restrictions on workflow.

\section{SPECIFIC WORKFLOW CONSIDERATIONS}

\section{Before Scanning}

Nuclear medicine and radiology technologists must have a good understanding of both imaging modalities. Extensive crosstraining was required when we started our trimodality PET/CTMR imaging. The most challenging task in a hybrid PET/MR imaging environment is operating the MR scanner with its nearly unlimited choice of pulse sequences and field-of-view coverage options. Patient preparation for PET/MR imaging and PET/CT is essentially identical. For the PET portion, the patient's dietary condition must be confirmed, and the level of serum glucose must be measured. Protocols for diabetic patients must be adhered to. Pregnancy must be ruled out and radiotracer uptake times must be standardized. For MR imaging, contraindications must be identified, including non-MR imaging-compatible implants and pacemakers. Patient positioning is identical to that for a stand-alone normal-bore $(60 \mathrm{~cm}) \mathrm{MR}$ imaging system (no wide-bore PET/MR imaging system is available), which may limit the ability to position patients with lung tumors in a standard arms-up position. The wider bore $(70 \mathrm{~cm})$ of an MR or PET/CT-MR imaging scanner is certainly one of the advantages of such a sequential system.

\section{General Protocol Aspects and Whole-Body PET/MR Imaging}

Simultaneous PET/MR Imaging studies require different considerations from sequential studies.

Sequential PET/CT-MR Imaging. This form of PET/MR imaging offers the advantage that it can be run in a sequential hybrid mode

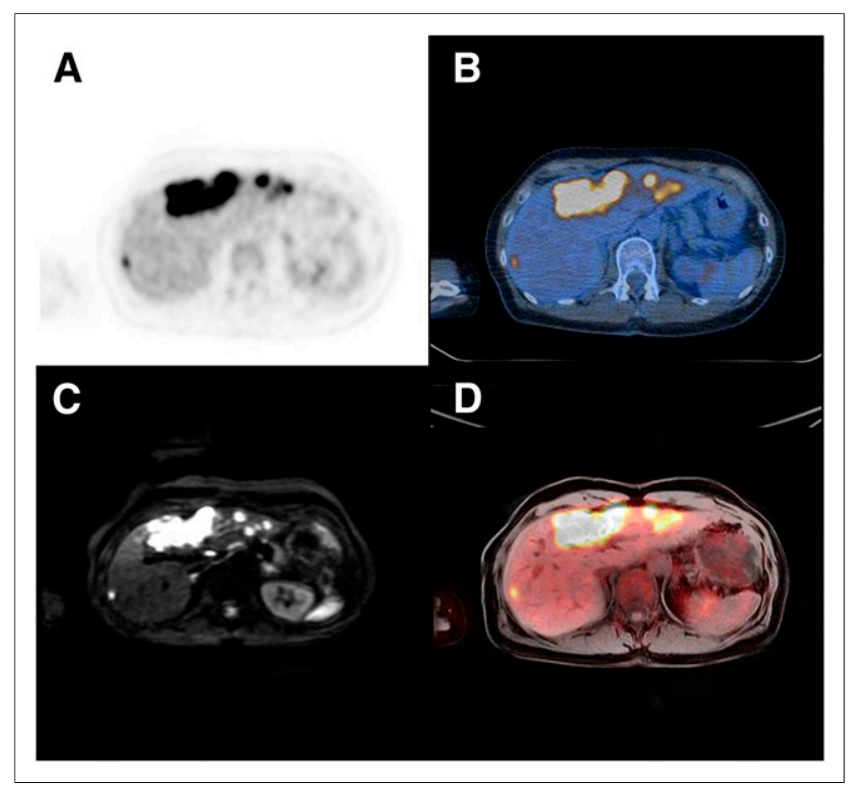

FIGURE 2. Patient with disseminated liver metastases in both liver lobes: PET only (A); PET/CT (B); diffusion-weighted MR imaging (C); $\mathrm{PET} / \mathrm{MR}$ imaging (D). Liver metastases are clearly seen on PET/CT as well as on PET/MR imaging. MR imaging also shows lesions in both liver lobes but does not contribute to diagnostic accuracy. 
that provides combined PET/CT-MR imaging data or as separate PET/CT and MR imaging systems (Fig. 3). Thus, MR imaging time does not have to be as carefully planned as with simultaneous PET/ MR imaging, at least if the systems are not run in a strict pipeline mode. Ideally, in a pipeline scenario, MR imaging (of a PET/CTMR imaging system) is performed during the tracer uptake time, allowing 30-45 $\mathrm{min}$ for MR imaging. After that, the patient can be moved to the PET/CT (trimodality system) portion of the examination.

Assuming a 30-min MR imaging time would then translate into the following: $0 \mathrm{~min}$ : ${ }^{18} \mathrm{~F}-\mathrm{FDG}$ injection, $25 \mathrm{~min}$ : uptake and placing of the patient in the MR scanner, 30-60 min: MR scanning of the patient, 60-90 min: PET/CT scanning of the patient, injection of the second patient at $30 \mathrm{~min}$, and so forth. This approach offers the advantage that the patient does not stay longer in the department than with a standard PET/CT procedure. Furthermore, attenuation-correction data are obtained from $\mathrm{CT}$ and not from MR imaging. However, when imaging and scheduling efficiency is the highest goal, the sequential PET/CT-MR imaging and simultaneous PET/MR imaging face the same challenge: The MR time should not substantially exceed the PET/CT time because otherwise PET/CT would wait in this setting for patients to be transferred from MR imaging.

Simultaneous PET/MR Imaging. The workflow for simultaneous PET/MR imaging starts with the standard ${ }^{18} \mathrm{~F}-\mathrm{FDG}$ uptake period of 60 min, followed by the simultaneous PET/MR imaging acquisition. Note that a whole-body PET scan requires approximately $30 \mathrm{~min}$ or less for completion, whereas special MR imaging sequences may require much longer imaging times. Thus, diligent choices for MR imaging pulse sequences are required. Several attenuation-correction approaches should probably be used for different body regions $(4,5)$. For the head, atlas techniques are available that are based on standard imaging sequences, which

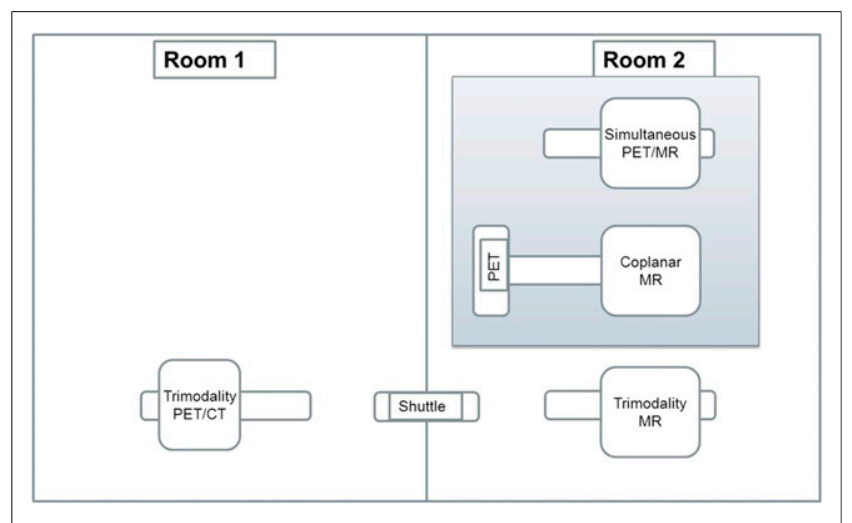

FIGURE 3. Schematic of three available PET/MR imaging solutions. Simultaneous PET/MR imaging is a one-room solution in which PET and MR imaging are acquired simultaneously (top row). Coplanar PET/MR imaging (middle row) incorporates stand-alone MR imaging that is connected to a PET-only system by a rotatable bed in the middle. No simultaneous imaging is possible; system can be occupied by one patient, and no PET/CT can be performed. These two systems (gray background) are one-room systems. Trimodality PET/CT-MR imaging incorporates stand-alone MR imaging system that is connected with fully clinical PET/CT system by a detachable shuttle system that fits into both systems (bottom row). No simultaneous imaging is possible; system can be used as coupled PET/CT-MR imaging (pipeline mode) or as independent PET/CT and MR imaging. This system is a two-room system. then compare the acquired images with a database for attenuation correction. For the rest of the body, the current technique, which is applicable for the head as well, is based on breath-hold, 2-point Dixon sequences from which image regions of different tissue classes (air, water, lung, soft tissue) are identified. For the attenuation correction of the patient's arms, which may be cropped in some MR pulse sequences, Dixon-based MR imaging or PET emission data are used. This approach is not satisfactory for the attenuation correction of bony regions (6).

Because PET is performed in a step-and-shoot mode-imaging is acquired with predefined scanning times and anatomic positions-MR data acquisition must be adapted to those anatomic positions to efficiently provide data for attenuation correction. Before the attenuation-correction scan, which frequently is done as the initial MR pulse sequence, the usual prescanning for localizing and shimming must be performed. However, because protocol and timing optimization is one of the highest priorities in clinical PET/MR imaging, it is desirable to develop smart PET/ MR imaging-adapted protocols. Prescanning can be completed in a short time (e.g., a normal prescan is $40 \mathrm{~s}$ for the first station and then only $5 \mathrm{~s}$ for the other stations), providing that the key MR imaging parameters are identical for every anatomic position. Depending on patient size, this can use 2 min of MR scanning time per patient. Current scanning ranges for attenuation correction (using a Dixon-based approach) are adapted to the approximately $25-\mathrm{cm}$ axial field of view of the PET scanners, with an overlap of 5-6 cm between scanning positions. Particularly for the head and the extremities, other pulse sequences such as ultrashortor zero-echo-time sequences for visualizing bone are possibly more appropriate for attenuation correction (7-9). However, a trade-off exists between the technically more advanced and more reliable sequences and the additional scanning times these sequences require.

Another issue is the development and use of smart attenuationcorrection protocols. Attenuation correction is usually done axially or coronally using body coils, which offers the advantage of a relatively quick whole-body survey that achieves good homogeneity across the field of view. However, these data do not provide sufficient diagnostic information compared with standard unenhanced PET/CT (10). Therefore, it would be desirable to perform the attenuation-correction scan with surface coils to obtain, first, data for attenuation correction and, second, a whole-body survey of diagnostic quality. Using this approach, time for the otherwise diagnostically insufficient attenuation-correction scan can be saved. Techniques for the correction of intensity inhomogeneities have been published (11). Continuous table motion is feasible in PET/MR imaging (12). Such an approach could possibly save time, especially when whole-body images are acquired. However, no straightforward technique exists for converting this approach into a reasonable PET/MR imaging workflow for larger patient cohorts.

\section{Current Literature}

Published protocols most frequently adapt the PET emission time to the required MR imaging time per anatomic position (13). This MR imaging-driven approach for protocol optimization leads to the predominant use of PET/MR imaging scanners for MR imaging. For instance, protocols in prostate cancer are aimed at optimizing the diagnostic information gained from MR imaging. Dual-modality systems are one imaging modality; thus, efficient combined protocols must be defined on the basis of the clinical 
need and indication. Several reports have used lengthy protocols that cover the whole body in full diagnostic quality, with contrast medium and several whole-body sequences (13-15). Particularly in prostate cancer, such protocols reflect an excessive bias toward MR imaging. However, in PET/MR imaging, the PET component is always available, so redundant information potentially obtainable by MR imaging can be omitted. In prostate cancer, PET/MR imaging is used to identify local recurrence, pelvic and retroperitoneal lymph node metastases, and bone metastases. Thus, a PET/ MR imaging protocol should be adapted to these goals. Therefore, it is not necessary to invest significant MR imaging time in dynamic contrast-enhanced imaging of the liver or respiration-gated sequences of the lung.

Publications reporting short and efficient protocols are few $(16,17)$. Kohan et al. (17) have shown that PET/MR imaging with just one attenuation-correction sequence for lymph node staging in patients with suspected or known lung cancer is only slightly inferior to $\mathrm{N}$ staging by PET/CT. This fact emphasizes the important role of PET for lymph node staging. It thus appears unlikely that PET/MR imaging provides diagnostic information in addition to that provided by PET/CT for lymph node assessment. That PET/ MR imaging is not inferior to PET/CT has also been reported for the staging of lung cancer. Another study by Schwenzer et al. (16) showed that contrast-enhanced PET/MR imaging was not inferior to PET/CT for staging of bronchial carcinoma. Here, only 3 sequences (one attenuation-correction and two diagnostic sequences) were used. Although the latter studies use a PET/MR imaging rather than MR imaging-driven workflow, they unfortunately provide clinical protocols of limited usefulness. Standard PET/CT oncology protocols should provide not only $\mathrm{N}$ staging but also $\mathrm{T}$ and $\mathrm{M}$ staging information. M staging especially is one of the major strengths of PET/CT, and it is expected to be important in PET/MR imaging as well. For example, in staging bronchial carcinoma, brain MR imaging to rule out metastases is recommended as early as stage IIB. Thus, dedicated brain MR imaging would have to be included in a clinically relevant PET/MR imaging protocol in this setting. Various clinical PET/MR imaging protocols are discussed in the next section.

\section{WORKFLOW PROPOSALS AND CONCEPTS}

Overall, 5 main clinical indications can be identified for simultaneous PET/MR imaging and sequential PET/MR imaging. First, as in PET/CT, oncology indications for staging, restaging, and therapy follow-up will likely play a prominent part in clinical PET/MR imaging. Second and third indications are cardiac imaging and neuroimaging, including neurooncology and neurodegenerative diseases. The fourth and fifth indications are pediatric imaging and musculoskeletal imaging. Pediatric imaging is important, particularly because of the achievable radiation dose reduction afforded by the new highly sensitive PET scanners placed inside the MR scanners in simultaneous PET/MR imaging and also, to a lesser degree, because of the replacement of CT with MR imaging. However, from a quantitative point of view, potential patient volumes are small compared with the first, second, and third applications. Musculoskeletal applications are potentially interesting in PET/MR imaging. However, PET is infrequently used for nononcologic musculoskeletal indications. Moreover, the relevant musculoskeletal MR coils are not routinely available for PET/MR imaging. Therefore, only the first 3 indications will be discussed further.

\section{Oncology}

The general differences between simultaneous and sequential PET/MR imaging have been discussed in the preceding text. Planning workflows for sequential PET/MR imaging is somewhat less critical than for simultaneous PET/MR imaging.

For the oncology applications, basic and advanced protocols must be differentiated. Although numerous protocols are available in clinical routines for single-modality MR imaging, only a few scenarios actually cover most indications in multimodality imaging. A good overview of fully diagnostic protocols that are not adapted to the specific PET/MR imaging setting has been provided by Martinez-Möller et al. (13). The efficiency of advanced protocols must be optimized. In addition to the basic protocol in a specific body compartment, high-resolution and contrast-enhanced MR imaging based on the clinical indication should be performed only if it does not require excessive time, in which case it would be better performed as single-modality MR imaging.

Organ-Based Protocols. Protocols are more straightforward when patients present with disease that is localized to a single organ or body region. In those cases, only localized PET/MR imaging confined to that body compartment might be needed to complete overall staging or to confirm suspected metastases (Fig. 4). Those protocols are especially easy to perform because they cover only one or two bed positions in PET/MR imaging and thus do not need special workflow planning or major restrictions on MR imaging time, and because whole-body PET can be performed even with a slightly prolonged MR acquisition time.

Basic Whole-Body Protocols. In whole-body oncology imaging, the clinical goals of PET/MR imaging are nearly identical to those of standard low-dose and unenhanced PET/CT. A basic protocol covers the whole body from the head to the proximal thighs. Thus, MR pulse sequences can be limited to whole-body transaxial T1- and T2-weighted images and at least one triggered pulse sequence for the chest and upper abdomen (18). Such a protocol offers the same overall diagnostic accuracy as unenhanced PET/CT, even for lung lesions. Any additional MR pulse sequences

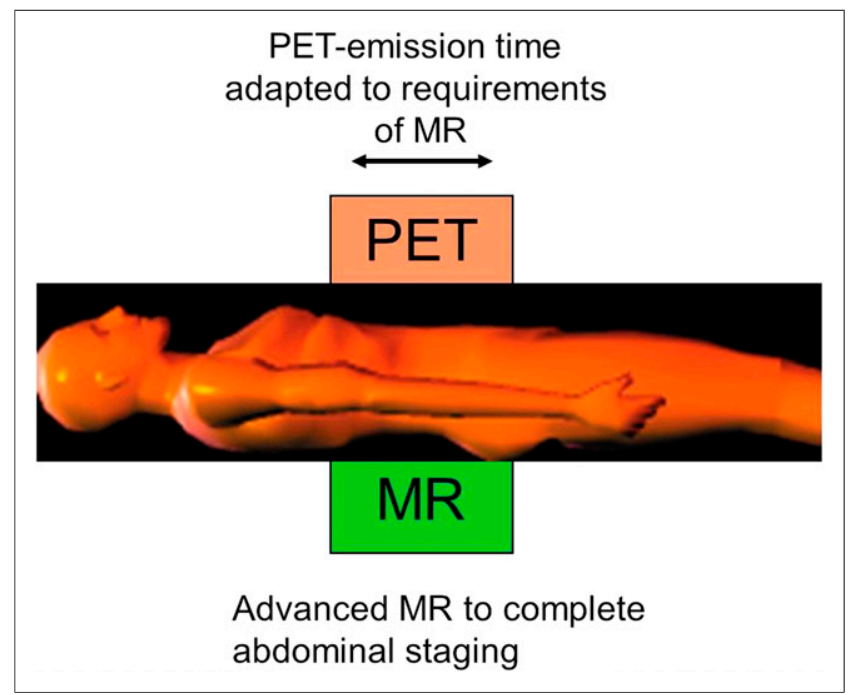

FIGURE 4. Schematic of possible PET/MR imaging partial-body data acquisition. In patient in whom previous partial-body imaging is available, PET/MR imaging might be applied in only one or two positions. PET imaging time then can be adapted to required MR imaging time. 
would be redundant or confirmatory but would not add diagnostic information. Such a protocol can be completed in 15-17 min (Fig. 5). The drawback of this approach is that fewer chest lesions will be detected. However, most missed lesions are small and of uncertain diagnostic relevance. Assuming standard PET emission scan durations of 2 min per bed position, truly simultaneous PET and MR imaging acquisitions would not be possible even for such short MR imaging protocols. Only T1- (Dixon) and T2-weighted sequences for attenuation correction and anatomic localization, but no additional triggered lung sequence, would be feasible in this time frame (Fig. 5). However, respiration-gated PET, which requires longer acquisition times, may offer improved diagnostic accuracy for lung assessments. Such an approach would allow longer imaging times for the MR sequences $(19,20)$, which can be accomplished using the same triggering device for PET and MR acquisitions (Fig. 5), which in turn enables optimization of both simultaneous data acquisition and equipment use.

Advanced Whole-Body Protocols. Advanced MR imaging protocols using high resolution and contrast enhancement in a specific body region can be performed. For such protocols, MR images could be acquired as follows: the whole-body attenuation scan would be followed by diagnostic whole-body imaging to obtain T1and T2-weighted images. For head and neck cancer protocols, axial T1- and T2-weighted images, diffusion-weighted images, and contrast-enhanced T1-weighted images may have to be acquired in multiple planes. Such advanced regional MR imaging would require approximately 35-40 min for completion. During the MR acquisition, dedicated high-resolution, quality PET images could be acquired ( $\sim 20 \mathrm{~min}$ ) (Fig. 6). However, this option is suboptimal because the MR imaging data acquisition requires much more time than the PET protocol. In therapy monitoring, the scenario is different. The key information is derived from PET; thus, a basic PET/ MR imaging protocol would frequently suffice. Initial literature data indicate that diffusion-weighted MR imaging in addition to PET provides redundant information (21), which is consistent with our own experience in patients with head and neck cancer. Other proposed protocols-for example, acquiring additional whole-body contrast-enhanced T1-weighted images at the end to exploit the

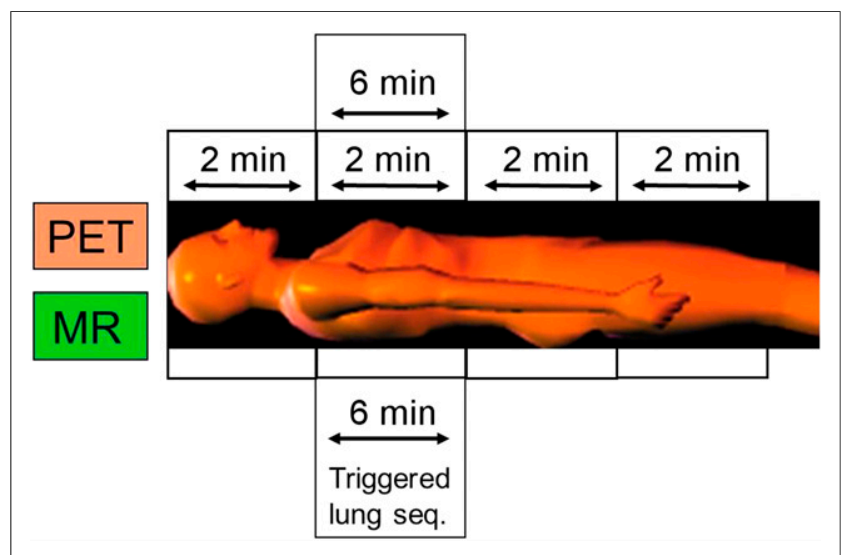

FIGURE 5. Schematic of possible basic PET/MR imaging partial-body data acquisition with triggered lung imaging. In MR imaging, Dixon T1weighted and coronal T2-weighted fast spin-echo images would be acquired in 2 min per bed position. Additionally, triggered lung sequence can be acquired, ideally with triggered PET acquisition (additional 4-6 $\mathrm{min}$ ). Such a protocol would still be acquired in 15-17 $\mathrm{min}$ and thus be comparable to standard PET/CT acquisition.

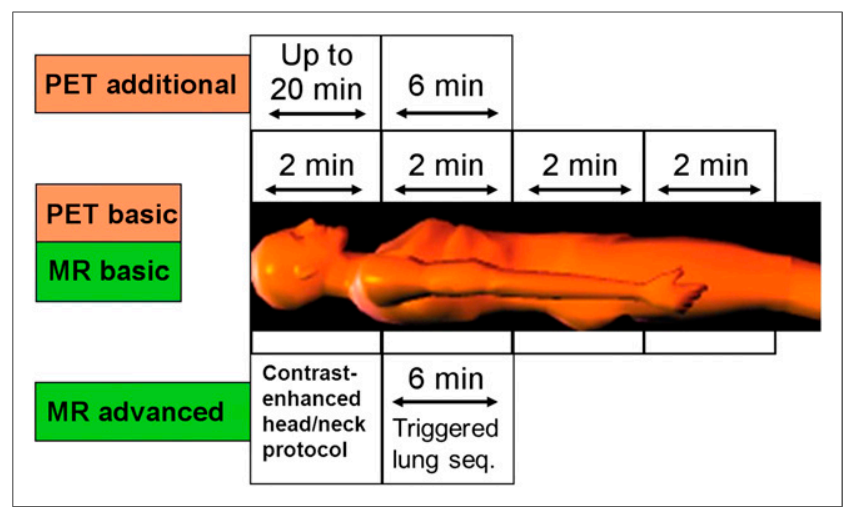

FIGURE 6. Schematic of possible advanced PET/MR imaging partialbody data acquisition with triggered lung imaging and additional contrast-enhanced MR imaging in one or two body compartments. This additional MR imaging acquisition typically adds up to 20-25 min of imaging time. One must decide whether this should be simultaneous with PET or MR imaging alone.

contrast medium that has already been injected-may fall into the same category: the additional MR information obtained is most probably only confirmatory.

Advanced abdominal and thoracic protocols are facing similar or even greater challenges, For instance, a gated MR imaging sequence is used for lung assessment, and dynamic contrastenhanced sequences are used for liver imaging. The timing issue becomes critical when gadolinium-EOB-DTPA is used for the detection and characterization of liver lesions. Protocols could be more efficient by adding a contrast-enhanced "rule out brain metastasis" protocol with an imaging time of approximately $20 \mathrm{~min}$. Such a protocol would keep the overall imaging time in the range of 40 min. For research comparisons between PET/CT and PET/ MR imaging, a quick attenuation-correction scan could be performed at the end of the procedure. In such a scenario, PET/MR imaging can be done first within the ${ }^{18} \mathrm{~F}$-FDG uptake time, and the PET imaging time points between PET/CT and PET/MR imaging are offset by only 10-15 min. However, such a protocol does not solve the previously mentioned workflow problems in clinical routines.

\section{Brain and Cardiac Applications}

Brain tumor imaging frequently requires both PET and MR imaging. Radiolabeled imaging probes of amino acid transport are more frequently used than ${ }^{18} \mathrm{~F}$-FDG. Although these PET procedures usually have shorter uptake periods than ${ }^{18} \mathrm{~F}$-FDG PET, the PET acquisition may contain a dynamic component and may therefore require $30 \mathrm{~min}$ for completion, as in the case of ${ }^{18} \mathrm{~F}$ fluoro-ethyl-L-tyrosine. In this case, the PET protocol allows 30 min of simultaneous MR imaging because the bed position is fixed. MR imaging can be started at the beginning of the radiotracer uptake period, and image acquisition can be extended until the PET acquisition is completed.

Nevertheless, practical and efficient MR brain imaging protocols are necessary-for instance, when assessing neurodegenerative diseases. Such studies using ${ }^{18}$ F-FDG or an amyloid-targeting PET probe use a single bed position, and images are acquired for 10-15 min 30-60 min after tracer injection. Some authors have advocated dynamic scanning, which requires an overall imaging time of approximately $60 \mathrm{~min}$. The major clinical impact of MR 
imaging in this setting is to rule out vascular disorders or other causes of dementia rather than to positively identify Alzheimer disease. Here, the protocol considerations discussed in the previous section apply. Other potential brain imaging indications for PET/MR imaging are under development.

For cardiac applications, PET with ${ }^{18}$ F-FDG is used for assessing myocardial viability. A single-bed-position PET emission scan can be acquired in 10-15 min. However, the major potential application of cardiac PET/MR imaging is quantitative myocardial perfusion imaging, which, with the currently available tracers $\mathrm{NH}^{3}$ and rubidium, requires dynamic imaging with the patient at rest and during stress. Completion of such protocols requires 40$75 \mathrm{~min}$. MR myocardial perfusion data are unlikely to provide additional information. Thus, the use of integrated PET/MR imaging for perfusion imaging appears to be of limited clinical value.

\section{CONCLUSION}

Current proposed PET/MR imaging protocols are mainly biased toward MR imaging protocol considerations and neglect the strength of integrated PET/MR imaging. Integrated protocols must be optimized to provide the most comprehensive diagnostic information. Deriving such information may not require the application of numerous MR pulse sequences. Furthermore, new MR pulse sequences are needed to match the clinical needs of PET/ MR imaging. These include pulse sequences of the ultrashort- or zero-echo-time type that provide improved visualization of bones and lung parenchyma. Moreover, improved pulse sequences suitable for MR-based attenuation correction, perhaps also ultrashortor zero-echo-time sequences, are desirable. Appropriate basic and advanced protocols as discussed here should aid in the improved use and application of PET/MR imaging. PET/MR imaging will remain a research tool for the foreseeable future to identify its true advantages over other imaging approaches. Advantages of the more expensive simultaneous systems compared with sequential (e.g., trimodality) systems need to be established. Early clinical studies suggest that PET/MR imaging is useful in brain imaging (oncology and neurodegenerative diseases), head and neck imaging, liver and gastrointestinal imaging, and gynecologic imaging.

\section{DISCLOSURE}

Patrick Veit-Haibach received IIS grants from Bayer Healthcare, Siemens Medical Solutions, GE Healthcare, and Roche Pharma, and speaker's fees from GE Healthcare. Gustav von Schulthess is a grant recipient from GE Healthcare and received speaker's fees from GE Healthcare. No other potential conflict of interest relevant to this article was reported.

\section{REFERENCES}

1. von Schulthess GK, Burger C. Integrating imaging modalities: what makes sense from a workflow perspective? Eur J Nucl Med Mol Imaging. 2010;37:980-990.

2. Veit-Haibach P, Kuhn FP, Wiesinger F, Delso G, von Schulthess G. PET-MR imaging using a tri-modality PET/CT-MR system with a dedicated shuttle in clinical routine. MAGMA. 2013;26:25-35.

3. Hany TF, Steinert HC, Goerres GW, Buck A, von Schulthess GK. PET diagnostic accuracy: improvement with in-line PET-CT system-initial results. Radiology. 2002;225:575-581.

4. Zaidi H. Is MR-guided attenuation correction a viable option for dual-modality PET/MR imaging? Radiology. 2007;244:639-642.

5. Hofmann M, Pichler B, Scholkopf B, Beyer T. Towards quantitative PET/MRI: a review of MR-based attenuation correction techniques. Eur J Nucl Med Mol Imaging. 2009;36(suppl 1):S93-S104.

6. Samarin A, Burger C, Wollenweber SD, et al. PET/MR imaging of bone lesions: implications for PET quantification from imperfect attenuation correction. Eur $J$ Nucl Med Mol Imaging. 2012;39:1154-1160.

7. Delso G, Carl M, Wiesinger F, et al. Anatomic evaluation of 3-dimensional ultrashort-echo-time bone maps for PET/MR attenuation correction. J Nucl Med. In press.

8. Keereman V, Mollet P, Berker Y, Schulz V, Vandenberghe S. Challenges and current methods for attenuation correction in PET/MR. MAGMA. 2013;26:81-98.

9. Weiger M, Brunner DO, Dietrich BE, Muller CF, Pruessmann KP. ZTE imaging in humans. Magn Reson Med. 2013;70:328-332.

10. Appenzeller P, Mader C, Huellner MW, et al. PET/CT versus body coil PET/ MRI: how low can you go? Insights Imaging. 2013;4:481-490.

11. Vovk U, Pernus F, Likar B. A review of methods for correction of intensity inhomogeneity in MRI. IEEE Trans Med Imaging. 2007;26:405-421.

12. Braun H, Ziegler S, Paulus DH, Quick HH. Hybrid PET/MRI imaging with continuous table motion. Med Phys. 2012;39:2735-2745.

13. Martinez-Möller A, Eiber M, Nekolla SG, et al. Workflow and scan protocol considerations for integrated whole-body PET/MRI in oncology. J Nucl Med. 2012;53:1415-1426.

14. Wetter A, Lipponer C, Nensa F, et al. Simultaneous ${ }^{18} \mathrm{~F}$ choline positron emission tomography/magnetic resonance imaging of the prostate: initial results. Invest Radiol. 2013;48:256-262.

15. Beiderwellen K, Gomez B, Buchbender C, et al. Depiction and characterization of liver lesions in whole body ${ }^{18}$ F-FDG PET/MRI. Eur J Radiol. 2013;82:e669-e675.

16. Schwenzer NF, Schraml C, Muller M, et al. Pulmonary lesion assessment: comparison of whole-body hybrid MR/PET and PET/CT imaging-pilot study. Radiology. 2012;264:551-558.

17. Kohan AA, Kolthammer JA, Vercher-Conejero JL, et al. N staging of lung cancer patients with PET/MRI using a three-segment model attenuation correction algorithm: initial experience. Eur Radiol. 2013;23:3161-3169.

18. Hüllner M, Appenzeller P, Kuhn FP, et al. PET/MR vs. PET/CT: comparison of whole body non contrast-enhanced imaging in oncologic staging and re-staging. Radiology. In press.

19. Würslin C, Schmidt H, Martirosian P, et al. Respiratory motion correction in oncologic PET using T1-weighted MR imaging on a simultaneous whole-body PET/MR system. J Nucl Med. 2013;54:464-471.

20. Guerra L, De Ponti E, Elisei F, et al. Respiratory gated PET/CT in a European multicentre retrospective study: added diagnostic value in detection and characterization of lung lesions. Eur J Nucl Med Mol Imaging. 2012;39:1381-1390.

21. Buchbender C, Hartung-Knemeyer V, Beiderwellen K, et al. Diffusion-weighted imaging as part of hybrid PET/MRI protocols for whole-body cancer staging: does it benefit lesion detection? Eur J Radiol. 2013;82:877-882. 